\title{
Intravenous leiomyomatosis extending from the inferior caval vein to the pulmonary artery
}

\author{
Giuseppe Virzì, MD, Salvatore Ragazzi, MD, Francesco Bussichella, MD, \\ Placido D'Agati, MD, Sergio Caputo, MD, Francesco Scaravilli, MD, \\ and Diego Piazza, MD, Catania, Italy
}

$\mathrm{I}$ ntravenous leiomyomatosis is a rare, benign, well-differentiated smooth muscle tumor seen exclusively in white women, primarily in those of postmenopausal age with a history of previous pregnancies and hysterectomy performed for a uterine leiomyoma, which may extend variable distances through the venous system and rarely metastasizes.

We present a case report of a 42-year-old white woman with cardiorespiratory symptoms determined by an intravascular leiomyoma extending from the inferior caval vein to the pulmonary artery.

\section{Clinical Summary}

The patient was a 42-year-old white woman. Her anamnesis was significant for myomectomy of multiple extrauterine leiomyomas in June 2001. In October 2003, she underwent a subtotal hysterectomy with a right salpingo-oophorectomy for recidive uterine leiomyoma involving the right ovary. In September 2004, she underwent a left ovariotomy for a large leiomyoma. In February 2005, she was admitted to the cardiology department with effort dyspnea, an unproductive cough, atypical thoracic pain, recurrent palpitations, and ankle swelling persisting after diuretic therapy. Echocardiography revealed the presence of a worm-like echoreflecting neoformation in the right atrium $(1.38 \times 1.25 \mathrm{~cm})$. Further ultrasonography, computed tomography, and magnetic resonance scan studies showed a large pelvic dishomogeneous tumor of approximately $18 \mathrm{~cm}$ in sagittal diameter, extending from the confluence of the common iliac veins to the inferior vena cava, crossing the right atrium and ventricle to the pulmonary artery (Figure 1). A combined operation to remove the intravascular and pelvic lesions was planned by the cardiology, cardiosurgery, and general surgery departments. In March 2005, resection of the intracardiac and intravascular lesion through a sternotomy under total circulatory arrest and hypothermia was successfully performed. The intravascular lesion was approximately $40 \mathrm{~cm}$ long and $9 \mathrm{~cm}$ wide (Figure 2). The pathologic examination of the specimen showed a leiomyoma with low cellularity. On immunochemistry, the

From the First Department of Surgery, Ospedale Vittorio Emanuele, Catania, Italy.

Received for publication Sept 28, 2006; accepted for publication Oct 24, 2006.

Address for reprints: Giuseppe Virzì, MD, Via Cimarosa 10, 95124 Catania (CT), Italy (E-mail: giuseppevirzi@ hotmail.com).

J Thorac Cardiovasc Surg 2007;133:831-2

$0022-5223 / \$ 32.00$

Copyright () 2007 by The American Association for Thoracic Surgery

doi:10.1016/j.jtcvs.2006.10.050

specimen showed the following: acute mono-

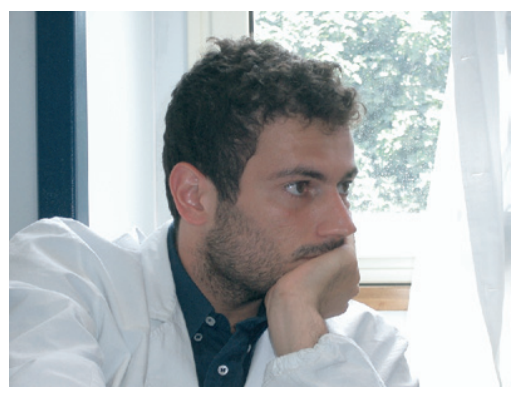

Dr Virzì cytic leukemia positivity, Ki67 positivity less than $3 \%$, and estroprogestinic receptor positivity in $75 \%$ of neoplastic cells. The postoperative course was uncomplicated, and the patient was moved to the cardiology department on the seventh day to have a vena caval filter inserted percutaneously through the femoral route and sited below the renal vein to prevent pulmonary embolization. Percutaneous embolization of both epigastric arteries was performed 2 days later to reduce the blood flow to the tumor. We admitted the patient to our department on the same day to perform a resection of the abdominal mass with a total hysterectomy. At laparotomy, a large, heterogeneous, and irregular pelvic mass arising from the uterine segment with abnormal vascular structures of various diameter and thickness was found. A tumoral resection with total hysterectomy was performed. The histopathologic examination showed a neoplasm, including a 5-cm-long uterine segment, constituted by isthmus and portio. The diagnosis of intravenous leiomyomatosis without malignant features was made. On immunochemistry, a high expression $(60 \%-70 \%)$ of estroprogestinic receptors was shown. The postoperative period was regular, and the patient was discharged on the ninth postoperative day.

Echocardiography, ultrasonography, and computed tomography studies performed postoperatively confirmed the absence of residual tumoral tissue. In view of the high detection of nuclear estradiol and progesterone receptors in the tumor, Tamoxifen treatment as an anti-estrogen was used to control the disease. The patient is alive and free of symptoms 17 months after the operation, without instrumental evidence of tumor recurrence. She has been scheduled for long-term follow-up.

\section{Discussion}

Intravenous leiomyomatosis is a rare, benign, smooth muscle tumor with unclear cause. Successful treatment depends on

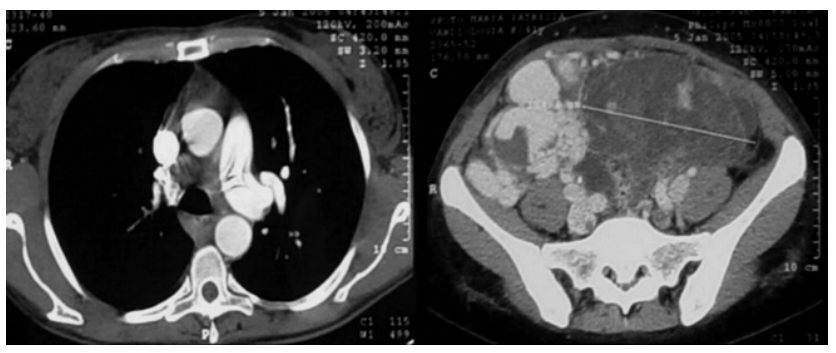

Figure 1. Computed tomography scans. Intravascular and intracardiac thrombus (left). Pelvic neoplasm (right). 


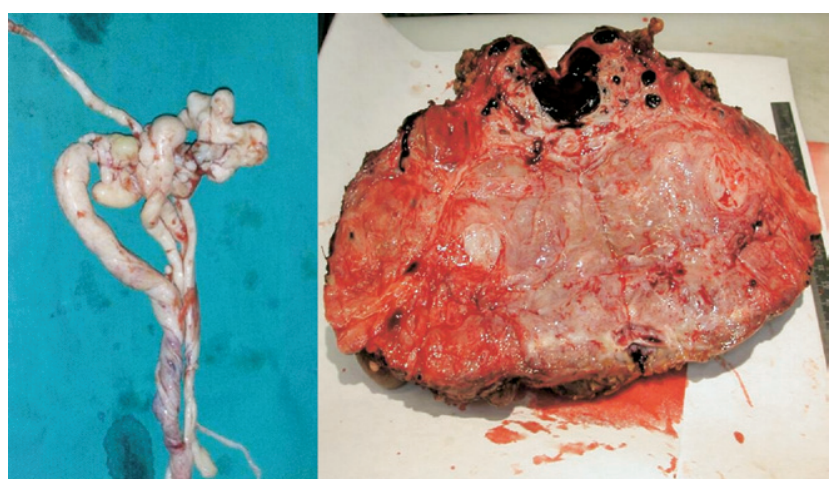

Figure 2. Intraoperative specimen. Intravenous leiomyomatosis extending into the right side of the heart, taking the shape of the inferior vena cava and right cavities (left). Abdominal tumor with abnormal vascular structures (right).

complete surgical tumor excision because recurrence is frequent despite the benign nature of the neoplasm, removal of ovarian function, and avoidance of postoperative estrogenic replacement therapy. ${ }^{1}$ Different surgical techniques have been proposed to approach intracardiac and abdominal neoplasms in the same or in separate operations. ${ }^{2}$ The detection of cytoplasmic estradiol and progesterone receptors in both the intravascular tumor and the metastasizing leiomyoma justify the use of an anti-estrogen, such as Tamoxifen, to control the disease progress. ${ }^{2}$ Because of the elevated incidence of recurrence $(30 \%)$ and the low metastatic potentiality, 3- and 6-month follow-ups after surgery are necessary. ${ }^{3}$

We stress the necessity of a pre-postoperative multidisciplinary approach to intravenous leiomyomatosis. We performed tumoral resection with a double-staged surgical strategy of cardiovascular and gynecologic operations to expose the patient to a lower postoperative bleeding risk. Hormonal therapy with Tamoxifen seems to be justified.

We thank Dr Tamburino Corrado, from the Department of Cardiology, Ospedale Ferrarotto, Catania, Italy; Dr Mignosa Carmelo, from the Department of Cardiosurgery, Ospedale S. Vincenzo, Taormina, Messina, Italy; and Drs Virzì Rosanna and Villari Loredana, from the Department of Pathology, Ospedale Vittorio Emanuele, Catania, Italy.

\section{References}

1. Steinmetz OK, Bedard P, Prefontaine ME, et al. Uterine tumor in the heart: intravenous leiomyomatosis. Surgery. 1996;119:226-9.

2. Lo KW-K, Lau T-K. Intracardiac leiomyomatosis. Case report and literature review. Arch Gynecol Obstet. 2001;264:209-10.

3. Evans AT 3rd, Symmonds RE, Gaffey TA. Recurrent pelvic intravenous leiomyomatosis. Obstet Gynecol. 1981;57:260-4.

\section{A different surgical approach for an intrathoracic expanding hematoma}

Kalliopi Athanassiadi, MD, ${ }^{a}$ Hans-Peter Reiffen, MD, PhD, ${ }^{\mathrm{b}}$ Nicolas Dickgreber, MD, PhD, ${ }^{\mathrm{c}}$ Florian Laenger, MD, PhD, ${ }^{\mathrm{d}}$ Christa-Maria Eschenbruch, MD, PhD, ${ }^{\mathrm{c}}$ Matthias Wilchelmi, MD, PhD, ${ }^{a}$ and Axel Haverich, MD, PhD, ${ }^{a}$ Hannover, Germany

hronic expanding hematomas are a rare clinical entity mimicking invasive soft-tissue neoplasms. We report the successful surgical treatment of a rare case of chronic expanding hematoma in the pleural cavity of an 83-year-old patient with a massive hemoptysis who was medically treated for tuberculosis 55 years earlier. A dissection of the

From the Departments of Thoracic and Cardiovascular Surgery, ${ }^{a}$ Anaesthesiology, ${ }^{\mathrm{b}}$ Pulmonology, ${ }^{\mathrm{c}}$ and Pathology, ${ }^{\mathrm{d}}$ Medical School of Hannover, Hannover, Germany.

Received for publication Sept 19, 2006; accepted for publication Oct 23, 2006.

Address for reprints: Kalliopi Athanassiadi, MD, Department for Thoracic and Cardiovascular Surgery, Medical School of Hannover, Carl-Neuberg Str. 1, 30625 Hannover, Germany (E-mail: kallatha@otenet.gr).

J Thorac Cardiovasc Surg 2007;133:832-4

$0022-5223 / \$ 32.00$

Copyright $\odot 2007$ by The American Association for Thoracic Surgery doi:10.1016/j.jtcvs.2006.10.049 calcified mass and extirpation of the hematoma were performed, the whole lung was expanded, the lobar and segmental arteries and bronchi were dissected, and the bronchial arteries were cauterized. In a follow-up of 4 months, the patient presented no recurrence, and a major operation (eg, a pleuropneumonectomy) was not necessary.

Chronic expanding hematomas are a rare clinical entity mimicking invasive soft-tissue neoplasms and producing mediastinal compression, usually in patients with a history of surgery for tuberculosis or tuberculous pleurisy. ${ }^{1,2}$

We report the successful surgical treatment of a rare case of chronic expanding hematoma in the pleural cavity of a patient with a massive hemoptysis who was medically treated for tuberculosis 55 years earlier.

\section{Clinical Summary}

An 83-year-old man was admitted to our department with hemoptysis and chest discomfort. He had a free medical history with the exception of tuberculosis 55 years ago. Chest radiography showed 